\title{
Identifying suitable models for the heat dynamics of buildings
}

\author{
Bacher, Peder; Madsen, Henrik
}

Published in:

Energy and Buildings

Link to article, DOI:

10.1016/j.enbuild.2011.02.005

Publication date:

2011

Document Version

Peer reviewed version

Link back to DTU Orbit

Citation (APA):

Bacher, P., \& Madsen, H. (2011). Identifying suitable models for the heat dynamics of buildings. Energy and Buildings, 43(7), 1511-1522. https://doi.org/10.1016/j.enbuild.2011.02.005

\section{General rights}

Copyright and moral rights for the publications made accessible in the public portal are retained by the authors and/or other copyright owners and it is a condition of accessing publications that users recognise and abide by the legal requirements associated with these rights.

- Users may download and print one copy of any publication from the public portal for the purpose of private study or research.

- You may not further distribute the material or use it for any profit-making activity or commercial gain

- You may freely distribute the URL identifying the publication in the public portal

If you believe that this document breaches copyright please contact us providing details, and we will remove access to the work immediately and investigate your claim. 


\title{
Identifying suitable models for the heat dynamics of buildings
}

\author{
Peder Bacher, Henrik Madsen \\ Informatics and Mathematical Modelling, Richard Pedersens Plads, Technical University of Denmark, Building 321, \\ DK-2800 Lyngby, Denmark
}

\begin{abstract}
The present paper suggests a procedure for identification of suitable models for the heat dynamics of a building. Such a procedure for model identification is essential for better usage of readings from smart meters, which is expected to be installed in almost all buildings in the coming years. The models can be used for different purposes, e.g. control of the indoor climate, forecasting of energy consumption, and for accurate description of energy performance of the building. Grey-box models based on prior physical knowledge and data-driven modelling are applied. This facilitates insight into otherwise hidden information about the physical properties of the building. A hierarchy of models of increasing complexity is formulated based on prior physical knowledge and a forward selection strategy is suggested enabling the modeller to iteratively select suitable models of increasing complexity. The performance of the models is compared using likelihood ratio tests, and they are validated using a combination of appropriate statistics and physical interpretation of the results. A case study is described in which a suitable model is sought after for a single storey 120 $\mathrm{m}^{2}$ building. The result is a set of different models of increasing complexity, with which building characteristics, such as: thermal conductivity, heat capacity of different parts, and window area, are estimated.
\end{abstract}

Keywords: Continuous time modelling, Likelihood ratio tests, Grey-box models, heat dynamics, thermal dynamics, buildings, model selection, lumped models, parameter estimation

Email address: pbac@dtu.dk (Peder Bacher)

$U R L$ : henrikmadsen.org (Henrik Madsen) 


\section{Contents}

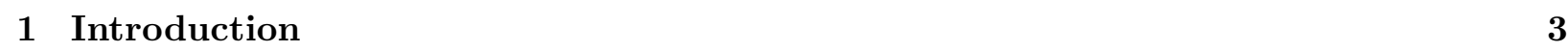

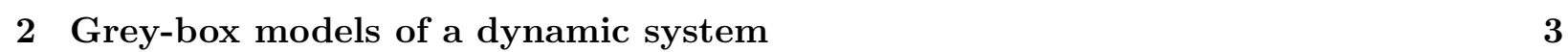

$2.1 \quad$ Maximum likelihood estimation of parameters . . . . . . . . . . . . . . 4

\begin{tabular}{|lll}
\hline & A statistical test for model selection & 5
\end{tabular}

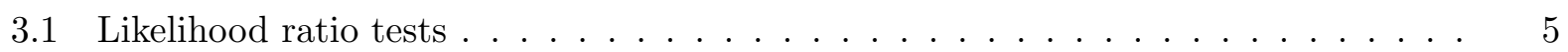

3.2 Forward selection . . . . . . . . . . . . . . . . . . . . . . . . 6

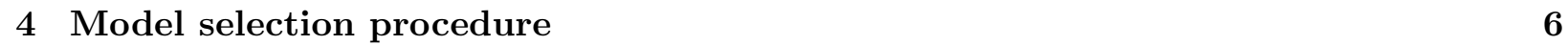

4.1 Model selection . . . . . . . . . . . . . . . . . . . . . . 6

4.2 Model evaluation $\ldots \ldots \ldots \ldots \ldots \ldots \ldots \ldots \ldots$

\begin{tabular}{|lll}
\hline 5 & Case study: model identification for a building & 8
\end{tabular}

5.1 Description of the building and measurement equipment . . . . . . . . . . . 8

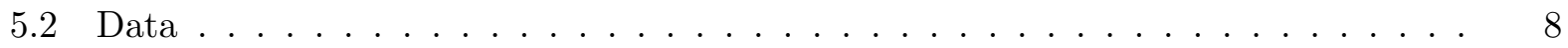

5.3 Applied models $\ldots \ldots \ldots \ldots \ldots \ldots$

5.3 .1 The full model TiTmTeThTsAeRia . . . . . . . . . . . . . . . . . 11

5.3 .2 The simplest model Ti . . . . . . . . . . . . . . . . . . 13

5.4 Model identification . . . . . . . . . . . . . . . . . . . . . . . . . . 14

5.5 Model evaluation . . . . . . . . . . . . . . . . . . . . . . . . . . 15

5.5 .1 Residuals . . . . . . . . . . . . . . . . . . . . 15

$5.5 .2 \quad$ Parameter estimates $\ldots \ldots \ldots \ldots \ldots \ldots$

\begin{tabular}{llr}
\hline 6 & Applications & 19
\end{tabular}

\begin{tabular}{lll}
\hline 7 & Conclusion & 20
\end{tabular} 


\section{Introduction}

This paper describes a new method for obtaining detailed information about the heat dynamics of a building based frequent readings of the heat consumption, indoor temperature, ambient air temperature, and other climate variables. Such a method is considered to be of uttermost importance as a key procedure for better usage of readings from smart meters, which is expected to be installed in almost all buildings in the coming years. The method is based on a procedure for selecting a suitable model of the heat dynamics for a building. Rabl (1988) gives an overview of techniques for steady state and for dynamic analysis of energy use in a building, the latter implicate modelling of the heat dynamics of the building. Such dynamic models can be realized with a set of differential equations, as carried out by Sonderegger (1978) and Boyer et al. (1996). Parameter estimation in dynamical models is known as system identification and a survey of different approaches for buildings is found in Bloem (1994). The applied models in the present method are grey-box models, which consist of a set of continuous time stochastic differential equations coupled with a set of discrete time measurement equations. Grey-box modelling is well proven as a comprehensive and accurate method to model dynamical systems and thereby obtain knowledge of the thermal properties of a building, see Madsen and Holst (1995), Andersen et al. (2000), and Jiménez et al. (2008). The problem of identifying a suitable model is both finding a model that is in agreement with the physical reality and finding a model, which has a complexity that is in agreement with the level of information embedded in data, which means that the model should neither be under-fitted nor over-fitted. The most suitable model is identified from a set of models of increasing complexity. A forward selection strategy is used, in which the modeller starts out with the simplest feasible model and iteratively selects models of increasing complexity. In each iteration the models are compared using likelihood-ratio tests and the models performances are evaluated. The selection procedure runs until no significant improvement of the model is found. See Pawitan (2001) and Madsen and Thyregod (2010) for an in-depth assessment of likelihood theory and model selection. The procedure is demonstrated by identifying a suitable model for a single storey $120 \mathrm{~m}^{2}$ building. The building is part of the experimental distributed energy system, Syslab, at Ris $\varnothing$ DTU in Denmark. It is constructed of wood on the outside and plaster boards on the inside, with a layer of insulation wool in between. The data used spans 6 days and stems from a set of experiments for building energy performance, which was carried out in the winter period of 2009. It is thoroughly described in Bacher and Madsen (2010).

The remaining of the article is organized as follows. The applied grey-box modelling technique is described in Section 2, and in Section 3 the statistical test used for model selection is described. Then the suggested procedure for identifying a suitable model is outlined in Section 4 . The following section is devoted to a case-study, where the procedure is applied. It starts with a description of the building and the data, followed by an outline of the applied models and the selection, and ends with a discussion of the results. Finally a perspective of the applications are given in Section 6 and the conclusions are drawn in Section 7 .

\section{Grey-box models of a dynamic system}

A grey-box model is established using a combination of prior physical knowledge and statistics, i.e. information embedded in data. The prior physical knowledge is formulated by a set of first-order stochastic differential equations, also called a stochastic linear state-space model in continuous time. The equations describe a lumped model of the heat dynamics of the building. It is emphasized 


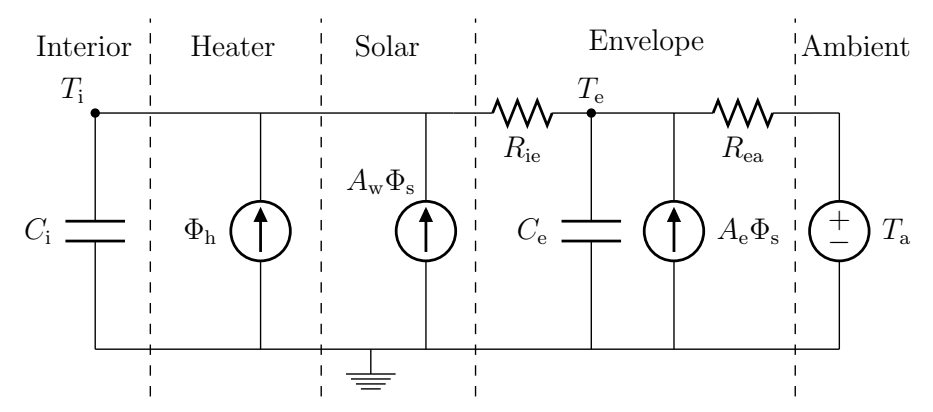

Figure 1: RC-network of the model described by Equation (1) and 22. The model is divided into different parts indicating the corresponding part of the building.

that the physical interpretation of the parameters is dependent on how the building is divided into entities in the model.

An example of a feasible model is given here. It has two state variables, one describing the interior temperature $T_{\mathrm{i}}$ and one representing the temperature of the building envelope $T_{\mathrm{e}}$. The first-order dynamics are represented by the stochastic differential equations

$$
\begin{aligned}
& d T_{\mathrm{i}}=\frac{1}{R_{\mathrm{ie}} C_{\mathrm{i}}}\left(T_{\mathrm{e}}-T_{\mathrm{i}}\right) d t+\frac{1}{C_{\mathrm{i}}} \Phi_{\mathrm{h}} d t+\frac{1}{C_{\mathrm{i}}} A_{\mathrm{w}} \Phi_{\mathrm{s}} d t+\sigma_{\mathrm{i}} d \omega_{\mathrm{i}} \\
& d T_{\mathrm{e}}=\frac{1}{R_{\mathrm{ie}} C_{\mathrm{e}}}\left(T_{\mathrm{i}}-T_{\mathrm{e}}\right) d t+\frac{1}{R_{\mathrm{ea}} C_{\mathrm{e}}}\left(T_{\mathrm{a}}-T_{\mathrm{e}}\right) d t+\sigma_{\mathrm{e}} d \omega_{\mathrm{e}}
\end{aligned}
$$

where $t$ is the time, $R_{\text {ie }}$ is the thermal resistance between the interior and the building envelope, $R_{\text {ea }}$ is the thermal resistance between the building envelope and the ambient air, $C_{\mathrm{i}}$ is the heat capacity of the interior, $C_{\mathrm{e}}$ is the heat capacity of the building envelope, $\Phi_{\mathrm{h}}$ is the energy flux from the heating system, $A_{\mathrm{w}}$ is the effective window area, $\Phi_{\mathrm{s}}$ is the energy flux from solar radiation, $T_{\mathrm{a}}$ is the ambient air temperature, $\left\{\omega_{\mathrm{i}, t}\right\}$ and $\left\{\omega_{\mathrm{e}, t}\right\}$ are standard Wiener processes, and $\sigma_{\mathrm{i}}^{2}$ and $\sigma_{\mathrm{e}}^{2}$ are the incremental variances of the Wiener processes. The model can be represented with the RC-network depicted in Figure 1, where the model is divided into different parts to show the corresponding parts of the building.

The physical model part is coupled with the data-driven model part with which the information embedded in observed data is used for parameter estimation. The data-driven part in the considered example is represented by the discrete time measurement equation

$$
Y_{k}=T_{\mathrm{i} k}+e_{k}
$$

where $k$ is the point in time $t_{k}$ of a measurement, $Y_{k}$ is the measured interior temperature and $e_{k}$ is the measurement error, which is assumed to be a Gaussian white noise process with variance $\sigma^{2}$. This assumption enables evaluation and tests of the performance of the model, since if it is met, this indicates that the physical model is consistent with the observed heat dynamics of the building.

\subsection{Maximum likelihood estimation of parameters}

Given a grey-box model, as described above, maximum likelihood estimates of the parameters can be obtained. Let the observations be represented by

$$
\mathcal{Y}_{N}=\left[Y_{N}, Y_{N-1}, \ldots, Y_{1}, Y_{0}\right]
$$


then the likelihood function is the joint probability density

$$
L\left(\theta ; \mathcal{Y}_{N}\right)=\left(\prod_{k=1}^{N} p\left(Y_{k} \mid \mathcal{Y}_{k-1}, \theta\right)\right) p\left(Y_{0} \mid \theta\right)
$$

where $p\left(Y_{k} \mid \mathcal{Y}_{k-1}, \theta\right)$ is a conditional density denoting the probability of observing $Y_{k}$ given the previous observations and the parameters $\theta$, and where $p\left(Y_{0} \mid \theta\right)$ is a parameterization of the starting conditions. The maximum likelihood estimates of the parameters are then found by

$$
\hat{\theta}=\arg \max _{\theta}\left\{L\left(\theta ; \mathcal{Y}_{N}\right)\right\}
$$

Due to the previously mentioned assumptions about the noise process and the fact that the model is linear, it follows that the conditional densities in Equation (6) are Gaussian densities. Since the conditional densities are Gaussian a Kalman filter can be used to calculate the likelihood function, and an optimization algorithm can be applied to maximize it, thereby calculating the maximum likelihood estimates, see Kristensen et al. (2004) for a detailed discussion. This is implemented in the computer software CTSM, which has been used for carrying out the parameter estimation, see more about the software at ${ }^{1}$ and in Kristensen and Madsen (2003).

\section{A statistical test for model selection}

Statistical tests can be utilized in the search for the most suitable model. If a model is a sub-model of larger model, then a likelihood ratio test can determine if the larger model performs significantly better than the sub-model. Using a sequence of such tests a strategy for selection of the best model can be evolved.

\subsection{Likelihood ratio tests}

Let a model have parameters $\theta \in \Omega_{0}$ where $\Omega_{0} \in \mathbb{R}^{r}$ is the parameter space and $r=\operatorname{dim}\left(\Omega_{0}\right)$ is the number of parameters in the model. Let a larger model have parameters $\theta \in \Omega$ where $\Omega \in \mathbb{R}^{m}$ and $\operatorname{dim}(\Omega)=m$, and assume that

$$
\Omega_{0} \subset \Omega
$$

i.e. the first model is a sub-model of the second model and $r<m$.

The likelihood ratio test

$$
\lambda\left(\mathcal{Y}_{N}\right)=\frac{\sup _{\theta \in \Omega_{0}} L\left(\theta ; \mathcal{Y}_{N}\right)}{\sup _{\theta \in \Omega} L\left(\theta ; \mathcal{Y}_{N}\right)}
$$

where $\mathcal{Y}_{N}$ is the observed values, can then be used to test the hypothesis

$$
H_{0}: \theta \in \Omega_{0} \quad \text { vs. } \quad H_{\mathrm{a}}: \theta \in \Omega \backslash \Omega_{0}
$$

since under $H_{0}$ the test statistic $-2 \log \left(\lambda\left(\mathcal{Y}_{N}\right)\right)$ converges to a $\chi^{2}$ distributed random variable with $(m-r)$ degrees of freedom as the number of samples in $\mathcal{Y}_{N}$ goes to infinity. If $H_{0}$ is rejected then the likelihood of the larger model is significantly higher than the likelihood of the sub-model, and it is concluded that $\mathcal{Y}_{N}$ is more likely to be observed with the larger model. Hence the larger model is needed over the sub-model to describe the information embedded in data. For more details see Madsen and Thyregod (2010).

\footnotetext{
1 www.imm.dtu.dk/ ctsm
} 


\subsection{Forward selection}

In a forward selection procedure the modeller starts with the smallest feasible model and then in each step extends the model with the part that gives the lowest p-value, i.e. the most significant improvement. The possible candidates for improvement that are selected in each iteration are the smallest possible extensions to the current model. The procedure stops when no extensions to the model yields a p-value below a pre-specified limit, usually set to $5 \%$.

\section{Model selection procedure}

Different strategies for identifying a suitable model is proposed in the literature and finding an appropriate strategy depends on the specific modelling setup. An purely algorithmic and exhaustive selection procedure is seldomly feasible, hence iterative methods, in which the modeller is partly involved in the selection, are commonly applied. Here, a forward selection procedure is suggested for identification of a suitable model for the heat dynamics. It is based on likelihood ratio testing, which is described in Section 3.1 .

\subsection{Model selection}

The procedure starts by a formulation of the simplest feasible model having parameter space $\Omega_{\mathrm{m}}$ and a full model with parameter space $\Omega_{\text {full }}$, such that

$$
\Omega_{\mathrm{m}} \subset \Omega_{\text {full }} .
$$

Within this range a set of models can be formed, in which a suitable model is to be identified. A suitable model is a sufficient model, which is the smallest model that describes all information embedded in the data (Madsen and Thyregod, 2010). The selection is initiated with the simplest model and then extensions of the model are iteratively added. The selection stops when all of the extensions to the selected model, gives a likelihood-ratio test p-value above the pre-specified limit. Hence the procedure will stop with a model from which no larger model can be found, with which it is significantly more likely to observe the data. As mentioned above a purely algorithmic procedure is not possible, hence the modeller must be involved to evaluate the models estimated in each iteration. The evaluation is carried out by analyzing the properties of residuals and parameter estimates, and if some of the properties are not in line with the assumptions and physical reality, then the modeller may have to influence the choice of model. The procedure is illustrated in Figure 2 and the steps are

Model fitting The models which are extended from the current model are fitted to the data by maximum likelihood estimation of the parameters.

Likelihood-ratio tests Calculate the likelihood-ratio test statistic for the current model versus each of the extended models. Stop if none of the tests have a p-value below $5 \%$ and use the current model as the final model, else then select the extended model which yield the lowest p-value.

Evaluate The modeller evaluates the selected extended model. If the result is satisfactory the model is kept and next iteration can be started; if not, the previous step should be repeated to select another extension. 


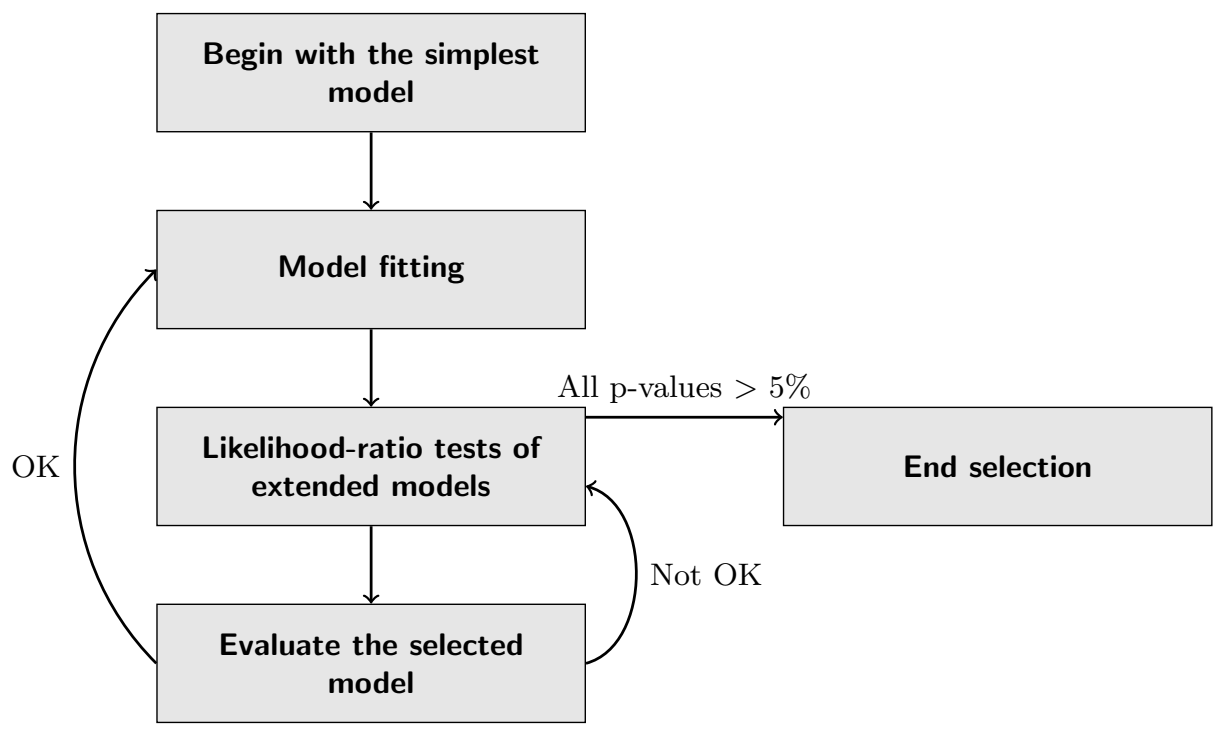

Figure 2: Illustration of the model selection procedure

If two extensions show an almost identical improvement, i.e. the p-values of the tests are nearly equal, the selection can be branched and extensions with different parts examined separately. The procedure will then end with several models, which cannot be tested directly against each other, and it is then up to the modeller to decide which should be preferred. This should be done by comparing the likelihoods, where if two models have almost equal likelihoods the smaller model should be preferred, and furthermore by an evaluation of the residuals and parameter estimates. It can also happen that several models have only marginal difference in performance and that each of them can be considered to be a sufficient model.

\subsection{Model evaluation}

In each step the selected model must be evaluated. This serves both to check if the model meet the assumptions and if it gives reasonable estimates from a physical point of view. Furthermore the evaluation can reveal model deficiencies from which it can be learned which parts of the model should be further elaborated. The evaluation should consist of the following:

- The assumption of white noise residuals should be inferred upon using the auto-correlation function $(\mathrm{ACF})$ and the cumulated periodogram $(\mathrm{CP})$, which can also reveal how well dynamics on different timescales are modelled.

- Plots of the inputs, outputs, and residuals. These plots can be used to understand which effects are not described properly by the model.

- Evaluation of the estimated physical parameters. Clearly the results should be consistent among different models, e.g. estimate of the thermal resistance of the building envelope should not change significantly among the models. Furthermore the modeller have to judge if the results are consistent with reality. 


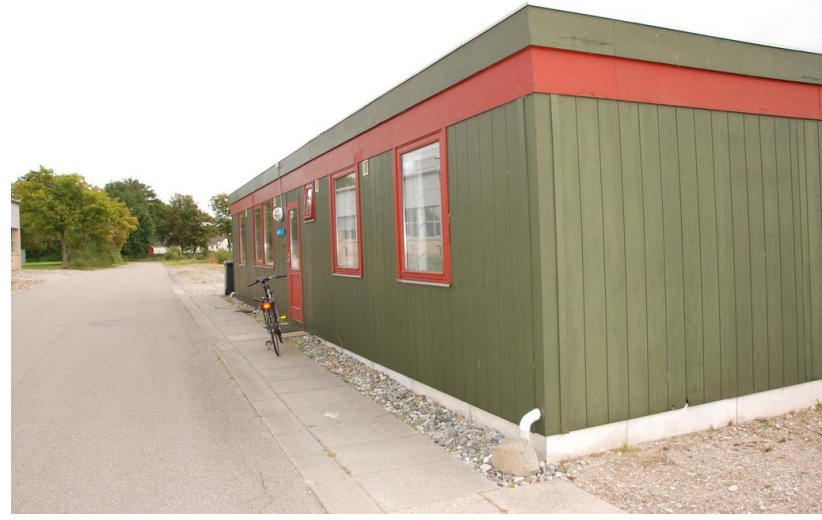

(a)

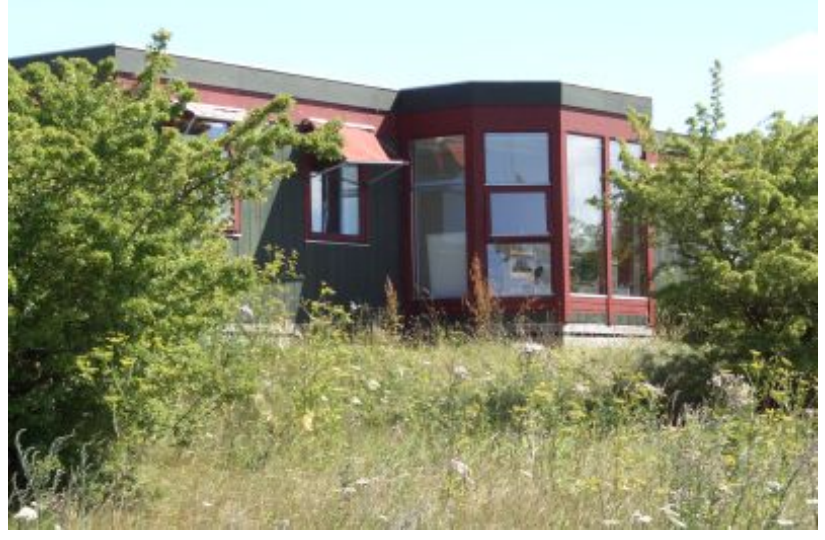

(b)

Figure 3: (a) the north facade and (b) is the south facade of the building.

\section{Case study: model identification for a building}

The method is demonstrated by applying it to identify a suitable model for a building. The building, named FlexHouse, is part of the experimental energy system Syslab, at Risø DTU in Denmark. It is well suited for such experiments since it has a controllable electrical heating system. Measurements consisting of five minute values over a period of six days are used, for further details see Bacher and Madsen (2010), in which a thorough description of the experiments and data is given. This section starts with a description of the building and measurement equipment, then the data is presented, followed by an outline of the considered models, and finally the model identification and evaluation is given.

\subsection{Description of the building and measurement equipment}

The outer walls of the building are constructed of wood on the outside and plaster boards on the inside, with a layer of insulation wool in between. An image of the north facade and an image of the south facade of the building can be seen in Figure 3. The building rests on piles, leaving an air gab between the ground and the building. The roof is flat and covered with roofing felt. The dimensions of the floor plan is approximately 7.5 times 16 meters. In Figure 4 the floor plan of the building is shown. A server system is installed in the building, which can control the electrical heaters located as indicated on the floor plan. To measure the indoor temperature Hobo U12-012 Temp/RH/Light/Ext sensors mounted on a small piece of wood was hanged freely in the middle of each each room. A small climate station is located two meters east of the building, the position relative to the building is indicated in Figure 4.

\subsection{Data}

The present study is based on data collected during a series of experiments carried out in February to April 2009. The following time series consisting of five minute average values are used:

$y\left({ }^{\circ} \mathrm{C}\right)$ A single signal representing the indoor temperature. It is formed as the first principal component of the measurements of the indoor temperature from the Hobo sensors. 


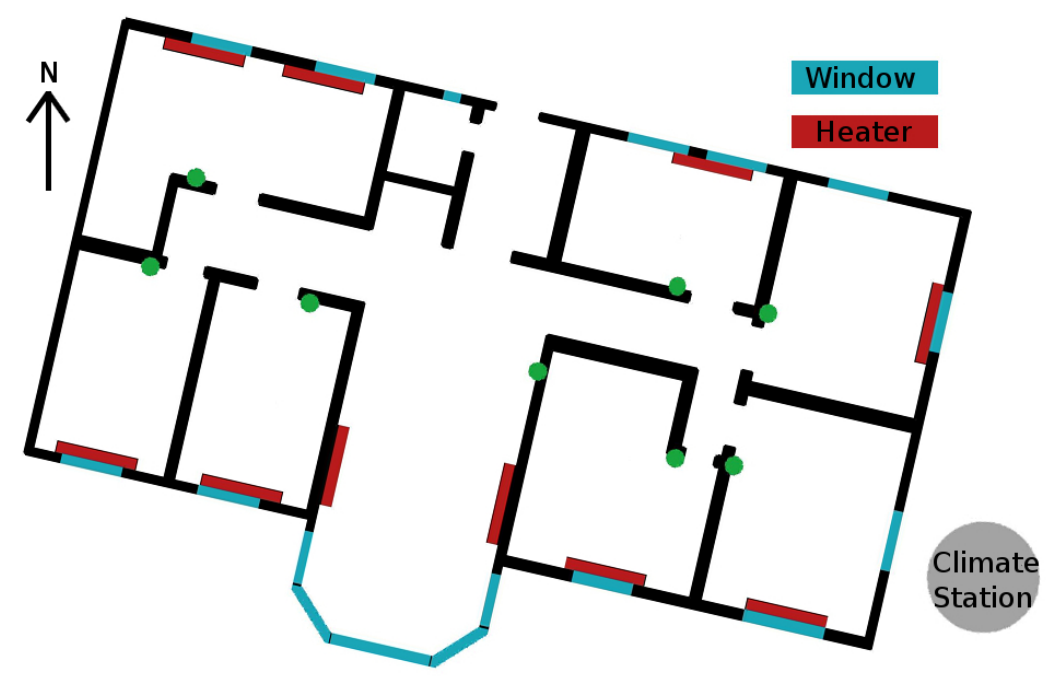

Figure 4: The floor plan of the building.

$T_{\mathrm{a}}\left({ }^{\circ} \mathrm{C}\right)$ Observed ambient air temperature at the climate station.

$\Phi_{\mathrm{h}}(\mathrm{kW})$ Total heat input from the electrical heaters in the building.

$\Phi_{\mathrm{s}}\left(\mathrm{kW} / \mathrm{m}^{2}\right)$ The global irradiance measured at the climate station.

Plots of the time series can be found in Figure 5. The controlled heat input is a pseudo-random binary sequence (PRBS), which has white noise properties and no correlation with the other inputs. It is designed to excite the heat dynamics at several ranges of frequencies in which the time constants of the building is expected to be, such that the information embedded in data is optimized for estimation of the heat dynamic properties of the building, see Madsen and Schultz (1993).

\subsection{Applied models}

The proposed procedure is such, that the modeller starts with the simplest model and iteratively selects more complex models. This implies fitting a set of models from the simplest model to the most feasible complex model, denoted the full model. In this section the set of applied models and the result of the iterative selection procedure is described. All the models are grey-box models, in which the physical part is stochastic linear state-space model (as presented in Section 2) and where the dynamics of the states can be written

$$
d \mathbf{T}=\mathbf{A} \mathbf{T} d t+\mathbf{B} \mathbf{U} d t+d \boldsymbol{\omega}
$$

where $\mathbf{T}$ is the state vector and $\mathbf{U}$ is the input vector, and none of the state variables or input variables are in $\mathbf{A}$ or $\mathbf{B}$ which only consist of parameters. All the considered models have an input vector with three inputs

$$
\mathbf{U}=\left[T_{\mathrm{a}}, \Phi_{\mathrm{s}}, \Phi_{\mathrm{h}}\right]^{T}
$$

All the models are lumped, but with a different structure, which implies that a given parameter does not necessarily represent the same physical entity in each model. For example the parameter 


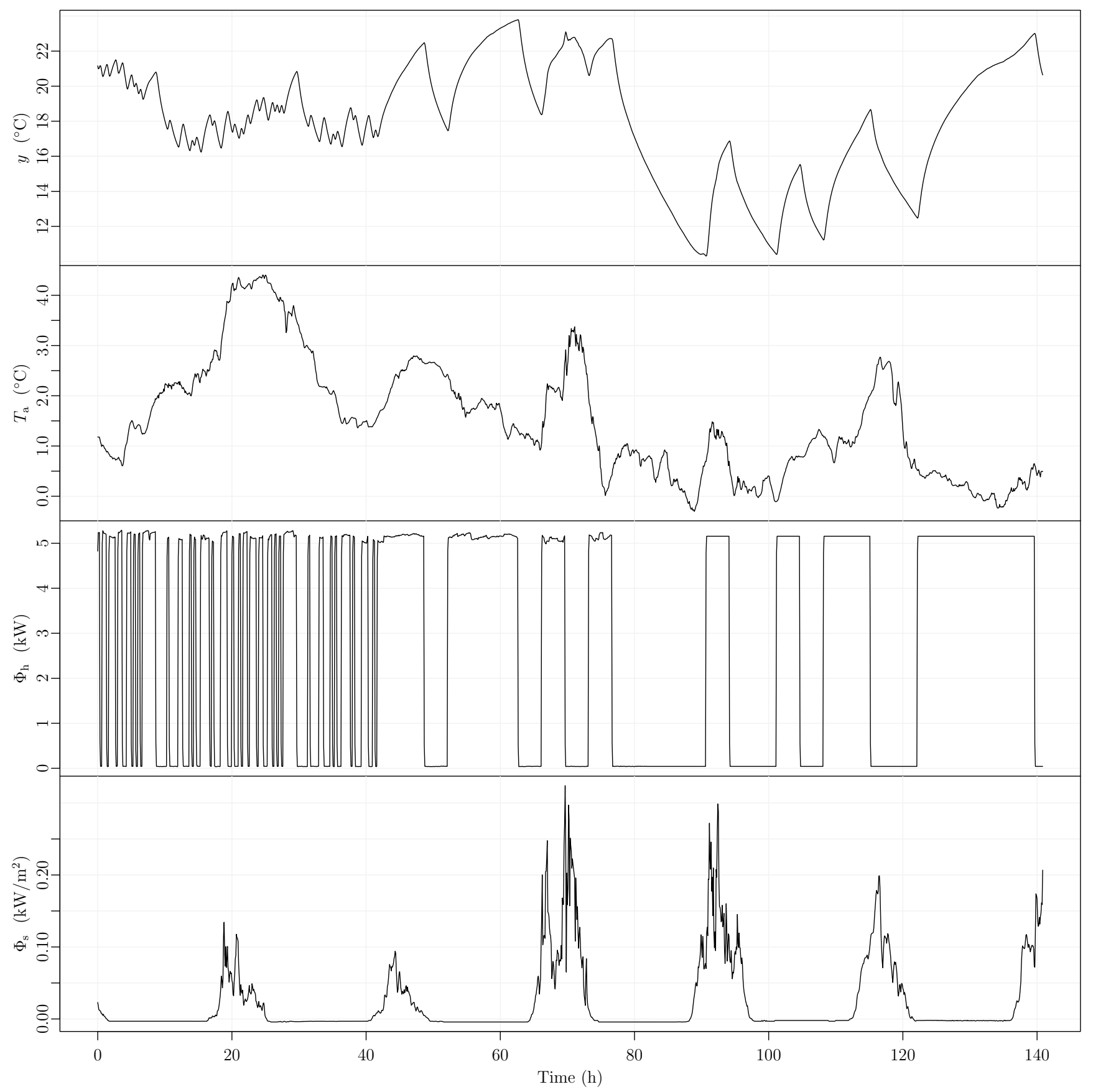

Figure 5: The data set. From the top, the first plot shows the observed indoor temperature $y$, the second shows the ambient air temperature $T_{\mathrm{a}}$, followed by a plot of the heat input $\Phi_{\mathrm{h}}$, and finally the lower plot shows the global irradiance $\Phi_{\mathrm{s}}$. 


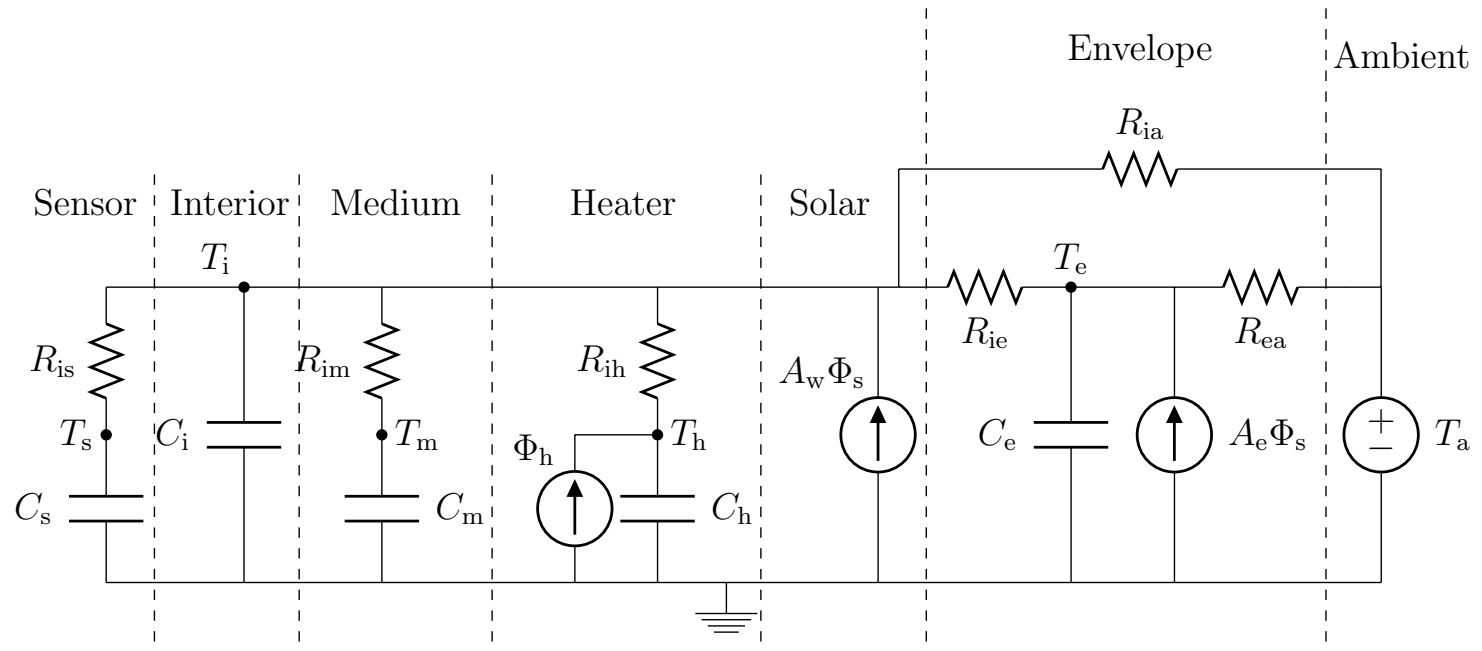

Figure 6: The full model TiTmTeThTsAeRia with the individual model parts indicated. This model includes all parts which is included in any of the applied models.

$C_{\mathrm{i}}$ is representing the heat capacity of the entire building in the simplest model, whereas this heat capacity is divided into five heat capacities in the full model, in which the parameter $C_{\mathrm{i}}$ represents the heat capacity of the indoor air. This is elaborated further in Section 5.5.2, where the parameter estimates for the models are presented. Furthermore it should be kept in mind that these models are linear approximations to the real system.

In the following sections the full and the simplest model are described, since they represent the range of applied models. First the full model is outlined to give a complete overview of all the individual parts, which is included in the models. Then the simplest model is presented, since it is the first model applied in the selection procedure and furthermore it illustrates how the models are lumped. Each model is named from its state vector and where needed a few parameter names. See 7 for a list of RC-networks corresponding to all applied models.

\subsubsection{The full model TiTmTeThTsAeRia}

The RC-network of the full model, which is the most complex model applied, is illustrated in Figure 6. This model includes all the individual parts of the building, which it is found feasible to include in linear models, with the current available data. The individual model parts are indicated on the figure. The model parts are:

Sensor The temperature sensors are modelled with a heat capacity and a thermal resistance to the interior.

Interior In the full model the interior is considered to be the indoor air (again remember that, since the models are lumped models, the building part represented by "Interior" is mostly different for each model) and it is modelled as a heat capacity connected to other parts by thermal resistances.

Medium A thermal medium inside the building is the interior walls and furniture, which is modelled with a heat capacity and a thermal resistance to the interior.

Heater The heaters are modelled by a heat capacity and a thermal resistance to the interior. 
Solar The heat input from solar radiation is modelled by the global irradiance multiplied with the effective window area.

Envelope The building envelope is modelled with a heat capacity and thermal resistances to both the interior and the ambient. A thermal resistance directly coupled to the ambient is also included.

Ambient The ambient is represented by the observed ambient air temperature.

The full model includes five state variables, that each represents the temperature in a part of the building, and they are:

$T_{\mathrm{s}}$ The temperature of the sensor, which for the full model is used as the model output, i.e. $Y_{k}$ in the measurement equation (Equation $(19)$ ).

$T_{\mathrm{i}}$ The temperature of the interior, i.e. the indoor air.

$T_{\mathrm{m}}$ The temperature of an interior thermal medium, i.e. interior walls and furniture.

$T_{\mathrm{h}}$ The temperature of the heaters.

$T_{\mathrm{e}}$ The temperature of the building envelope.

The parameters of the model represent different thermal properties of the building. This includes thermal resistances:

$R_{\text {is }}$ between the interior and the sensor,

$R_{\mathrm{im}}$ between the interior and the interior thermal medium,

$R_{\text {ih }}$ between the heaters and the interior,

$R_{\text {ia }}$ between the interior and the ambient,

$R_{\text {ie }}$ between from the interior and the building envelope,

$R_{\text {ea }}$ between the building envelope and the ambient.

The heat capacities of different parts of the building are represented by:

$C_{\mathrm{s}}$ for the temperature sensor,

$C_{\mathrm{i}}$ for the interior,

$C_{\mathrm{m}}$ for the interior walls and furniture,

$C_{\mathrm{h}}$ for the electrical heaters,

$C_{\mathrm{e}}$ for the building envelope.

Finally two coefficients are included, each representing an estimate of an effective area in which the energy from solar radiation enters the building. They are:

$A_{\mathrm{w}}$ The effective window area of the building. 


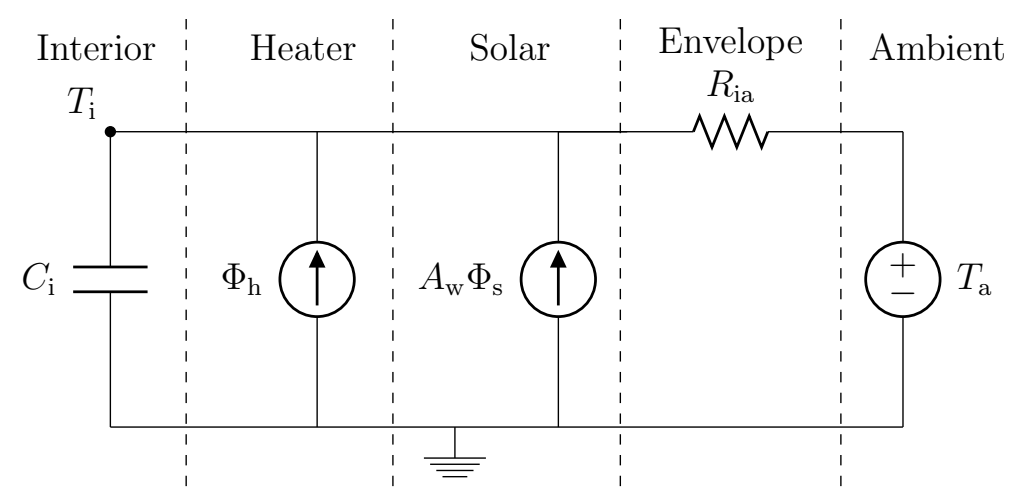

Figure 7: RC-network of the model $T i$, which is the simplest feasible model.

$A_{\mathrm{e}}$ The effective area in which the solar radiation enters the building envelope.

The set of stochastic differential equations describing the heat flows in the full model are

$$
\begin{aligned}
d T_{\mathrm{s}}= & \frac{1}{R_{\mathrm{is}} C_{\mathrm{s}}}\left(T_{\mathrm{i}}-T_{\mathrm{s}}\right) d t+\sigma_{\mathrm{s}} d \omega_{\mathrm{s}} \\
d T_{\mathrm{i}}= & \frac{1}{R_{\mathrm{is}} C_{\mathrm{i}}}\left(T_{\mathrm{s}}-T_{\mathrm{i}}\right) d t+\frac{1}{R_{\mathrm{im} C_{\mathrm{i}}}}\left(T_{\mathrm{m}}-T_{\mathrm{i}}\right) d t+\frac{1}{R_{\mathrm{ih}} C_{\mathrm{i}}}\left(T_{\mathrm{h}}-T_{\mathrm{i}}\right) \\
& \frac{1}{R_{\mathrm{ie}} C_{\mathrm{i}}}\left(T_{\mathrm{e}}-T_{\mathrm{i}}\right) d t+\frac{1}{R_{\mathrm{ia}} C_{\mathrm{i}}}\left(T_{\mathrm{a}}-T_{\mathrm{i}}\right) d t+\frac{1}{C_{\mathrm{i}}} A_{\mathrm{w}} \Phi_{\mathrm{s}} d t+\sigma_{\mathrm{i}} d \omega_{\mathrm{i}} \\
d T_{\mathrm{m}}= & \frac{1}{R_{\mathrm{im}} C_{\mathrm{m}}}\left(T_{\mathrm{i}}-T_{\mathrm{m}}\right) d t+\sigma_{\mathrm{m}} d \omega_{\mathrm{m}} \\
d T_{\mathrm{h}}= & \frac{1}{R_{\mathrm{ih}} C_{\mathrm{h}}}\left(T_{\mathrm{i}}-T_{\mathrm{h}}\right) d t+\frac{1}{C_{\mathrm{h}}} \Phi_{\mathrm{h}} d t+\sigma_{\mathrm{h}} d \omega_{\mathrm{h}} \\
d T_{\mathrm{e}}= & \frac{1}{R_{\mathrm{ie}} C_{\mathrm{e}}}\left(T_{\mathrm{i}}-T_{\mathrm{e}}\right) d t+\frac{1}{R_{\mathrm{ea}} C_{\mathrm{e}}}\left(T_{\mathrm{a}}-T_{\mathrm{e}}\right) d t+\frac{1}{C_{\mathrm{e}}} A_{\mathrm{e}} \Phi_{\mathrm{s}} d t+\sigma_{\mathrm{e}} d \omega_{\mathrm{e}}
\end{aligned}
$$

and the measurement equation is

$$
Y_{k}=T_{\mathrm{s}, k}+e_{k}
$$

since the observed temperature is encumbered with some measurement error.

\subsubsection{The simplest model $\mathrm{Ti}$}

The simplest model considered is illustrated by the RC-network in Figure 7. The model has one state variable $T_{\mathrm{i}}$ and the following parameters:

$R_{\mathrm{ia}}$ The thermal resistance from the interior to the ambient.

$C_{\mathrm{i}}$ The heat capacity of the entire building, including the indoor air, interior walls, furniture etc., and the building envelope.

$A_{\mathrm{w}}$ is the effective window area of the building. 


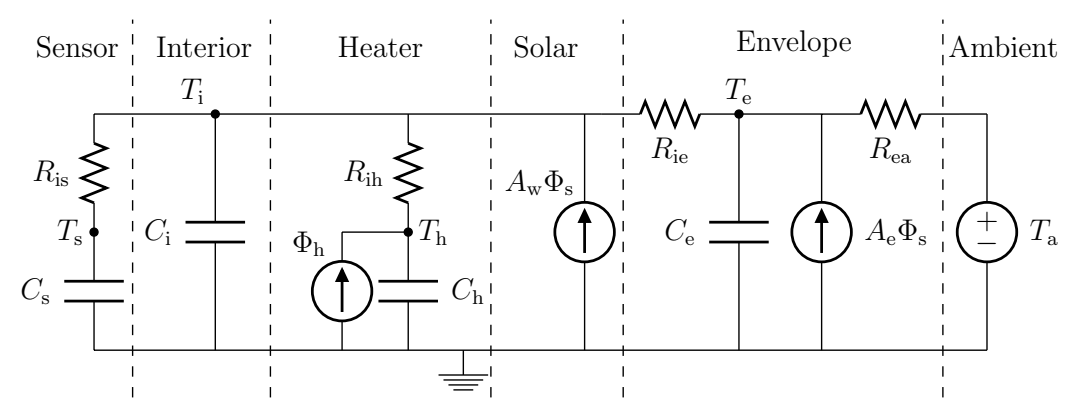

Figure 8: The final selected model TiTeThTsAe with the individual model parts indicated.

The stochastic differential equation describing the heat flow is

$$
\frac{d T_{\mathrm{i}}}{d t}=\frac{1}{R_{\mathrm{ia}} C_{\mathrm{i}}}\left(T_{\mathrm{a}}-T_{\mathrm{i}}\right)+\frac{1}{C_{\mathrm{i}}} A_{\mathrm{w}} \Phi_{\mathrm{s}}+\frac{1}{C_{\mathrm{i}}} \Phi_{\mathrm{h}}+\sigma_{\mathrm{i}} \frac{d \omega_{\mathrm{i}}}{d t}
$$

and the measurement equation is

$$
Y_{k}=T_{\mathrm{i}, k}+e_{k}
$$

Note the differences in representation of the building parts between the simplest and full model, e.g. $R_{\text {ia }}$ represent the thermal resistance of the building envelope in the simplest model, whereas this is represented by a coupling of $R_{\mathrm{ia}}, R_{\mathrm{ie}}$, and $R_{\mathrm{ea}}$ in the full model.

\subsection{Model identification}

The identification procedure is applied to find a sufficient model in the set of models ranging from $T i$ to TiTmTeThTsAeRia. The log-likelihood of each model, which is fitted, is listed in Table 1 ordered by the iterations of the model selection. The procedure begins with the simplest model. Then in the first iteration four extended models are fitted and TiTh is selected since it has the highest log-likelihood, hence the lowest p-value of the likelihood-ratio tests (the four models have the same number of parameters). The selection procedure is carried out until no significant extension can be found, which occurs in iteration number five. During each iteration the current selected model is evaluated, see Section 5.5. It is found that the models selected in each iteration are all satisfying the evaluation with respect to improvement of the results etc. In Table 2 the result of likelihood-ratio tests for model expansion in each iteration is listed. Clearly, the expansions carried out in the first three iterations indicate very significant improvements of the model. In iteration four, the improvement is still below $5 \%$, whereas no improvement is found in iteration five. The procedure thus ends with TiTeThTsAe as a sufficient model, which is illustrated by the RC-network in Figure 8 .

\subsection{Model evaluation}

In the following the selected models are evaluated as outlined in Section 4.2 .

\subsubsection{Residuals}

Plots of output, inputs, and residuals for each model can be seen in Figure 9. For each model the auto-correlation function (ACF) of the residuals is plotted in Figure 10 and the cumulated periodogram $(\mathrm{CP})$ in Figure 11. It is seen directly from the plot of the residuals from the simplest 


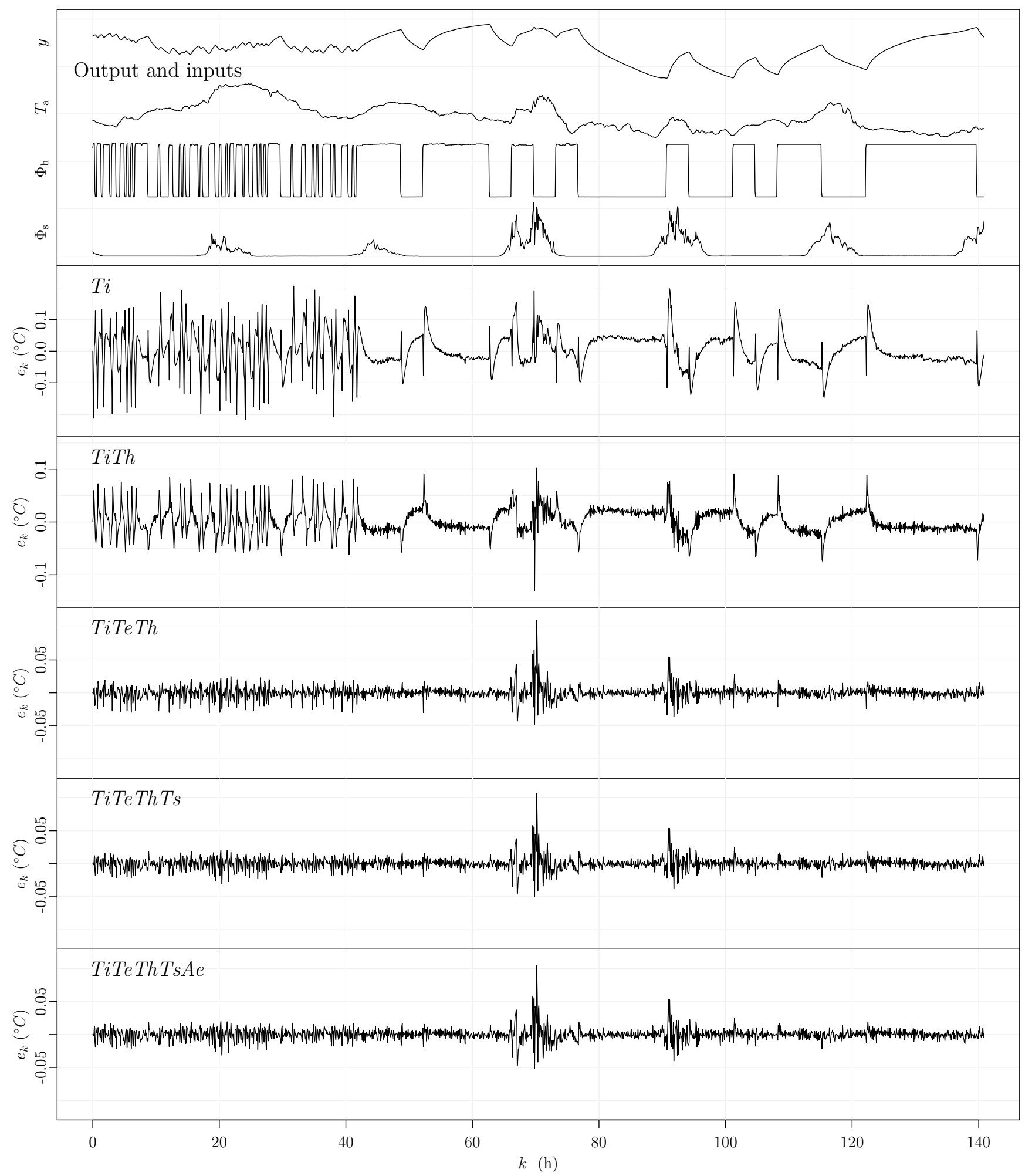

Figure 9: The upper plot is of the output and inputs, and the following plots are of the residuals for each of the selected models. On each plot of the residuals the model name is indicated. 

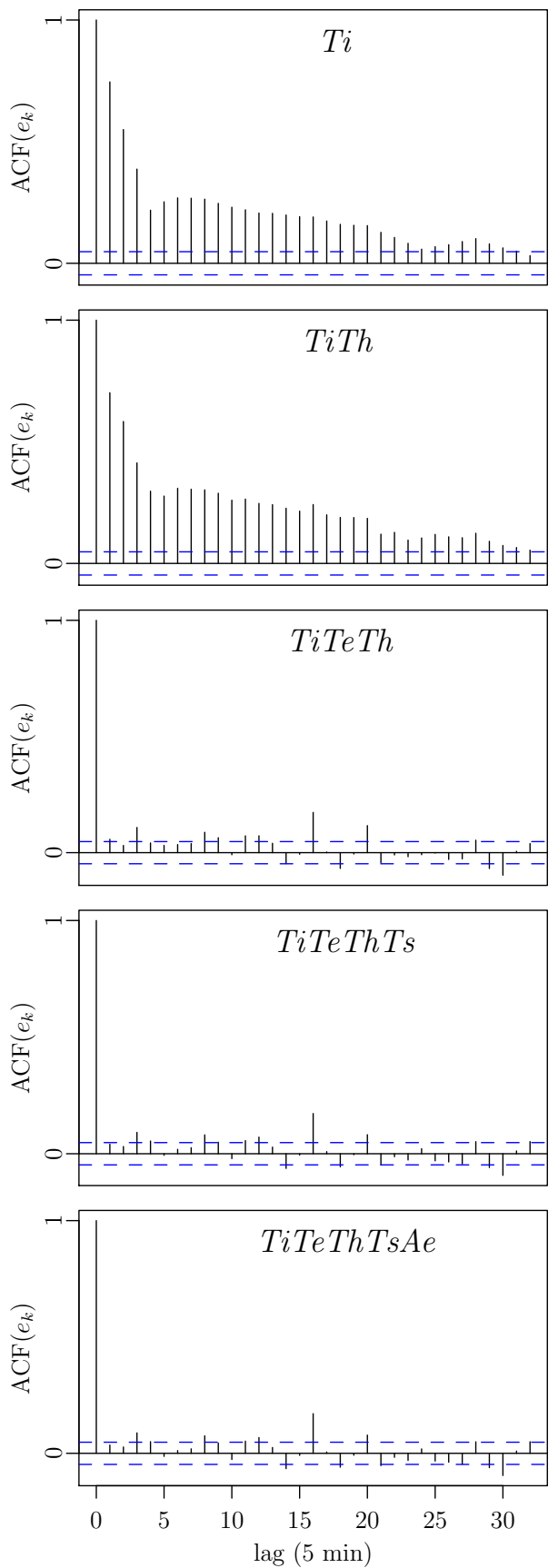

Figure 10: The auto-correlation function of the residuals for each of the selected models. 

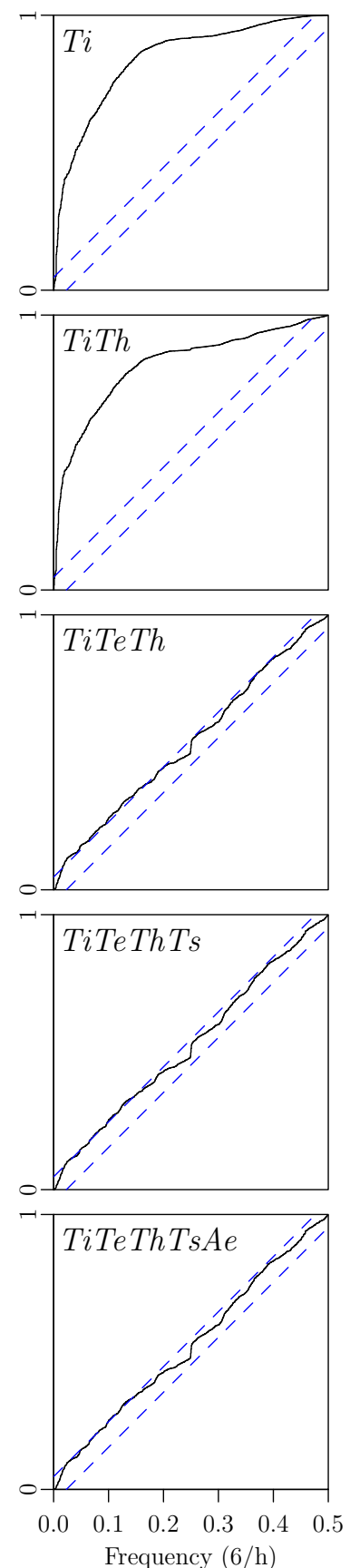

Figure 11: The cumulated periodogram of the residuals for each of the selected models. 


\begin{tabular}{|c|c|c|c|c|}
\hline Iteration & & Models & & \\
\hline $\begin{array}{l}\text { Start } \\
l\left(\theta ; \mathcal{Y}_{N}\right) \\
m\end{array}$ & $\begin{array}{c}T i \\
2482.6 \\
6\end{array}$ & & & \\
\hline 1 & $\begin{array}{c}\text { TiTe } \\
3628.0 \\
10\end{array}$ & $\begin{array}{c}\mathrm{TiTm} \\
3639.4 \\
10\end{array}$ & $\begin{array}{c}\text { TiTs } \\
3884.4 \\
10\end{array}$ & $\begin{array}{c}\text { TiTh } \\
3911.1 \\
10\end{array}$ \\
\hline 2 & $\begin{array}{c}\text { TiThTs } \\
4017.0 \\
14 \\
\end{array}$ & $\begin{array}{c}\text { TiTmTh } \\
5513.1 \\
14 \\
\end{array}$ & $\begin{array}{c}\text { TiTeTh } \\
5517.1 \\
14 \\
\end{array}$ & \\
\hline 3 & $\begin{array}{c}\text { TiTeThRia } \\
5517.3 \\
15\end{array}$ & $\begin{array}{c}\text { TiTeThAe } \\
5520.5 \\
15\end{array}$ & $\begin{array}{c}\text { TiTmTeTh } \\
5534.5 \\
18\end{array}$ & $\begin{array}{c}\text { TiTeThTs } \\
5612.4 \\
18\end{array}$ \\
\hline 4 & $\begin{array}{c}\text { TiTeThTsRia } \\
5612.5 \\
19\end{array}$ & $\begin{array}{c}\text { TiTmTeThTs } \\
5612.9 \\
22\end{array}$ & $\begin{array}{c}\text { TiTeThTsAe } \\
5614.6 \\
19\end{array}$ & \\
\hline 5 & $\begin{array}{c}\text { TiTmTeThTsAe } \\
5614.6 \\
23\end{array}$ & $\begin{array}{c}\text { TiTeThTsAeRia } \\
5614.7 \\
20\end{array}$ & & \\
\hline
\end{tabular}

Table 1: Log-likelihood $l\left(\theta ; \mathcal{Y}_{N}\right)$ for the fitted models ordered by iterations of the model selection procedure and in each row by log-likelihood. In each iteration the extended model with highest log-likelihood is selected, which is the rightmost models in the table. The number of estimated parameters for each model is indicated by $m$.

model $T i$, that they do not have white noise properties and that they are not independent of the inputs. The ACF of the residuals also clearly show a high lag dependency, and the CP reveals that the model is not detailed enough to describe the dynamics. Examining the plot of the residuals for the model selected in the first iteration, TiTh, it is seen that the level of the residuals is reduced compared to the residuals for Ti. The ACF and $\mathrm{CP}$ indicate that the assumption of white noise residuals is not fulfilled. From the plot of the residuals for the model selected in the second iteration, TiTeTh, it is seen that the level of the residuals is reduced dramatically, but that some dependency of the inputs is still seen, mostly from the solar irradiance. The ACF reveals that the characteristics of the residuals are much closer to white noise, which is also seen from the CP, indicating that the model now describes the heat dynamics of the building quite well. The plot of the residuals, $\mathrm{ACF}$, and $\mathrm{CP}$ for the model selected in the third iteration TiTeThTs, reveals only slight improvements compared to the previous model. Finally the plots for the final selected model TiTeThTsAe, show that almost no differences can be observed from the previous model. The highest level of error can be observed where the solar irradiance is high, hence it is found that further improvement of the model should be focused on the part in which the solar radiation enters the building, or alternatively in letting the incremental variance of the Wiener process depend on the solar radiation. 


\begin{tabular}{lccccc}
\hline Iteration & Sub-model & Model & $m-r$ & $-2 \log (\lambda(y))$ & p-value \\
\hline 1 & Ti & TiTh & 4 & 4121 & $<10^{-16}$ \\
2 & TiTh & TiTeTh & 4 & 4634 & $<10^{-16}$ \\
3 & TiTeTh & TiTeThTs & 4 & 274 & $<10^{-16}$ \\
4 & TiTeThTs & TiTeThTsAe & 1 & 6.4 & 0.011 \\
5 & TiTeThTsAe & TiTeThTsAeRia & 1 & 0.17 & 0.68 \\
\hline
\end{tabular}

Table 2: Tests carried out in the model selection procedure.

\subsubsection{Parameter estimates}

The parameter estimates of the selected models are evaluated in the following. The estimates are presented in Table 3 together with the time constants calculated for each of the selected models. The total heat capacity and thermal resistance of the building envelope estimated by the selected models are presented in Table 4. As found by evaluating the residuals, see previous section, the models $T i$ and TiTh do not describe the dynamics of the system very well, which implies that the estimates of the heat capacities are not reliable. Estimates of the heat capacities found by the tree larger models are more credible, especially it is seen that the time constants are almost equal, indicating that the models comprise the same dynamics. The exact physical interpretation of the smaller heat capacities $C_{\mathrm{i}}, C_{\mathrm{h}}$, and $C_{\mathrm{s}}$ cannot be given, but it is noted that their sum, for each of the three larger models, is quite close ranging from 1.03 to $1.08\left[\mathrm{kWh} /{ }^{\circ} \mathrm{C}\right]$.

The estimated total thermal resistance of the building envelope and thereby the UA-values is quite similar for all models, as seen in Table 4 .

\begin{tabular}{lccccc}
\hline Model & Ti & TiTh & TiTeTh & TiTeThTs & TiTeThTsWithAe \\
\hline$C_{\mathrm{i}}$ & 2.07 & 1.36 & 1.07 & 0.143 & 0.0928 \\
$C_{\mathrm{e}}$ & - & - & 2.92 & 3.24 & 3.32 \\
$C_{\mathrm{h}}$ & - & 0.309 & 0.00139 & 0.321 & 0.889 \\
$C_{\mathrm{s}}$ & - & - & - & 0.619 & 0.0549 \\
$R_{\mathrm{ia}}$ & 5.29 & 5.31 & - & - & - \\
$R_{\mathrm{ie}}$ & - & - & 0.863 & 0.909 & 0.897 \\
$R_{\mathrm{ea}}$ & - & - & 4.54 & 4.47 & 4.38 \\
$R_{\mathrm{ih}}$ & - & 0.639 & 93.4 & 0.383 & 0.146 \\
$R_{\mathrm{is}}$ & - & - & - & 0.115 & 1.89 \\
$A_{\mathrm{w}}$ & 7.89 & 6.22 & 5.64 & 6.03 & 5.75 \\
$A_{\mathrm{e}}$ & - & - & - & - & 3.87 \\
\hline$\tau_{1}$ & 10.9 & 0.16 & 0.129 & 0.0102 & 0.0102 \\
$\tau_{2}$ & - & 8.9 & 0.668 & 0.105 & 0.105 \\
$\tau_{3}$ & - & - & 18.4 & 0.786 & 0.788 \\
$\tau_{4}$ & - & - & - & 19.6 & 19.3 \\
\hline
\end{tabular}

Table 3: The estimated parameters. The heat capacities, $C_{\mathrm{x}}$, are in $\left[\mathrm{kWh} /{ }^{\circ} \mathrm{C}\right]$. The thermal resistances, $R_{\mathrm{xx}}$, are in $\left[{ }^{\circ} \mathrm{C} / \mathrm{kW}\right]$. The areas, $A_{\mathrm{x}}$, are in $\left[\mathrm{m}^{2}\right]$. The time constants, $\tau_{x}$, are in hours. Note that the physical interpretation for many of the parameters is different for each model. 


\begin{tabular}{lccccc}
\hline Model & Ti & TiTh & TiTeTh & TiTeThTs & TiTeThTsAe \\
\hline$C_{\text {total }}$ & 2.07 & 1.67 & 3.99 & 4.32 & 4.36 \\
$R_{\text {envelope }}$ & 5.29 & 5.31 & 5.40 & 5.38 & 5.28 \\
$\alpha_{\text {UA }}$ & 1.55 & 1.55 & 1.52 & 1.53 & 1.55 \\
\hline
\end{tabular}

Table 4: The total heat capacity $\left[\mathrm{kWh} /{ }^{\circ} \mathrm{C}\right]$ and thermal resistance $\left[{ }^{\circ} \mathrm{C} / \mathrm{kW}\right]$ of the building envelope estimated by the selected models. The UA-values $\alpha_{\mathrm{UA}}$ are in $\left[\mathrm{W} /\left({ }^{\circ} \mathrm{Cm}^{2}\right)\right]$.

\section{Applications}

Identification of a suitable model of the heat dynamics of a building based on frequent readings of heat consumption, indoor temperature, ambient air temperature, and climate variables, will be very useful for different purposes. Important fields of application are:

Accurate description of energy performance of the building An energy signature of buildings can provide important information for energy- and cost effective improvements of the building. The most effective actions to be taken for an individual building can be identified. Furthermore the heat consumption due to physical effects, such as a poor isolated building envelope, can be separated from behavioral effects, e.g. a high indoor temperature.

Forecasting of energy consumption for heating Forecasting of energy consumption for heating can be used for integration of large amounts of renewable energy, such as wind- and solar energy. Implementation of electrical heating with hot water tanks for heat storage in individual houses can be profitable in the near future. Knowledge of the heat dynamics of buildings is essential to forecasting and control of such systems.

Indoor climate control Control of the indoor temperature, ventilation etc. to provide a good indoor climate conditions can be carried out with methods which include models of the heat dynamics. The models can also be extended to include the effect of wind and thereby provide information of the air tightness of buildings.

\section{Conclusion}

A procedure for identification of the most suitable models for the heat dynamics of a building has been described and applied on the basis of data from an experiment carried out in February 2009. The procedure is based on likelihood-ratio testing combined with a forward selection strategy. The proposed models are grey-box models, where a combination of prior physical knowledge and data-driven modelling is utilized. The data used for the modelling consist of: climate data measured at the location, measurements of the indoor temperature, and a PRBS controlled heat input.

The results of the identification procedure are evaluated and discussed, both in a statistical and physical context. The evaluation reveal that the selected model meet the assumptions of white noise residuals, hence it can be applied to give reliable estimates consistent with reality and the results are statistically validated. Furthermore model deficiencies are pointed out, from which further advancement of the model should be pursued. For the considered building this is primarily on the model part where the solar radiation input enters the building.

It has been shown that the method is able to provide rather detailed knowledge of the heat dynamics of the building. This includes for instance the thermal resistance of the envelope and 
thereby the UA-value, parameters describing the capabilities for storing heat, and the time constants of the building.

Andersen, K. K., Madsen, H., Hansen, L. H., 2000. Modelling the heat dynamics of a building using stochastic differential equations. Energy and Buildings 31 (1), 13-24.

Bacher, P., Madsen, H., 2010. Experiments and data for building energy performance analysis : Financed by the danish electricity saving trust. Tech. rep., DTU Informatics, Building 321, Kgs. Lyngby.

Bloem, J. J. (Ed.), 1994. System Identification Applied to Building Performance Data. CEC-EUR 15885 EN.

Boyer, H., Chabriat, J., GrondinPerez, B., Tourrand, C., Brau, J., MAY 1996. Thermal building simulation and computer generation of nodal models. BUILDING AND ENVIRONMENT 31 (3), 207-214.

Jiménez, M., Madsen, H., Bloem, J., Dammann, B., 2008. Estimation of non-linear continuous time models for the heat exchange dynamics of building integrated photovoltaic modules. Energy \&amp; Buildings 40 (2), $157-167$.

Kristensen, N. R., Madsen, H., 2003. Continuous time stochastic modelling, CTSM 2.3 - mathematics guide. Tech. rep., DTU.

Kristensen, N. R., Madsen, H., Jørgensen, S. B., 2004. Parameter estimation in stochastic grey-box models. Automatica 40 (2), $225-237$.

Madsen, H., Holst, J., 1995. Estimation of continuous-time models for the heat dynamics of a building. Energy and Buildings 22 (1), 67-79.

Madsen, H., Schultz, J., 1993. Short time determination of the heat dynamics of buildings. Tech. rep., DTU.

Madsen, H., Thyregod, P., 2010. Introduction to General and Generalized Linear Models. CRC Press.

Pawitan, Y., 2001. In All Likelihood: Statistical Modelling and Inference Using Likelihood. Oxford University Press.

Rabl, A., 1988. Parameter-estimation in buildings - methods for dynamic analysis of measured energy use. Journal of Solar Energy Engineering-transactions of the Asme 110 (1), 52-66.

Sonderegger, R. C., 1978. Dynamic models of house heating based on equivalent thermal parameters. Ph.D. thesis, Princeton Univ., NJ. 
RC-networks of applied models 


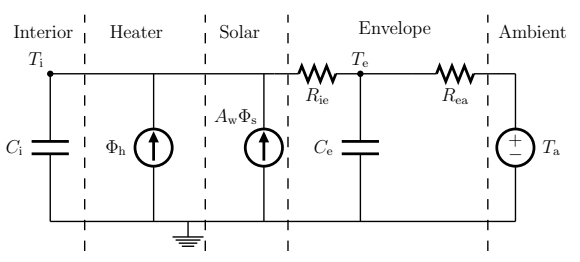

(a) RC-network network of TiTe.

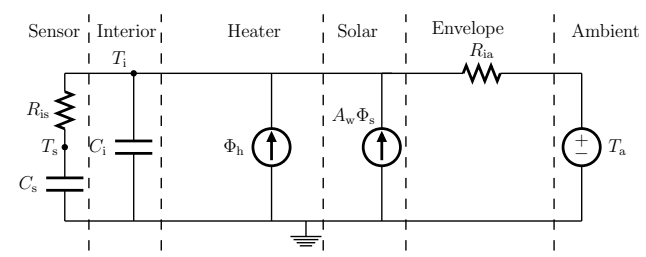

(c) RC-network network of TiTs.

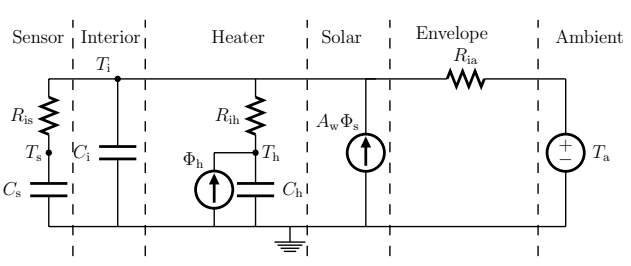

(e) RC-network network of TiThTs.

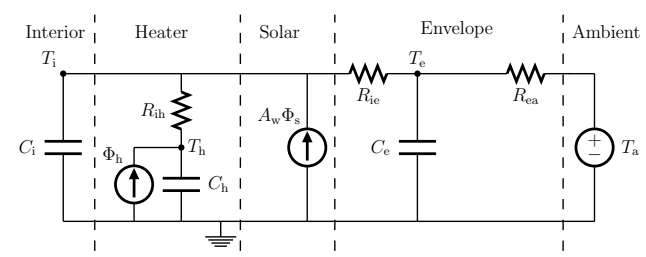

(g) RC-network network of TiTeTh.

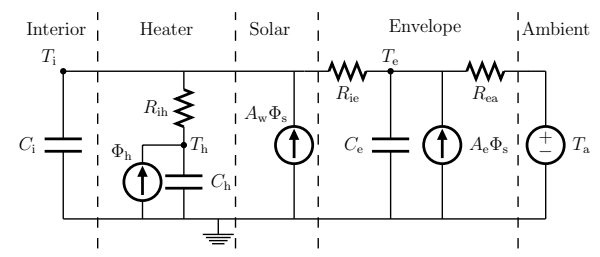

(i) RC-network network of TiTeThAe.

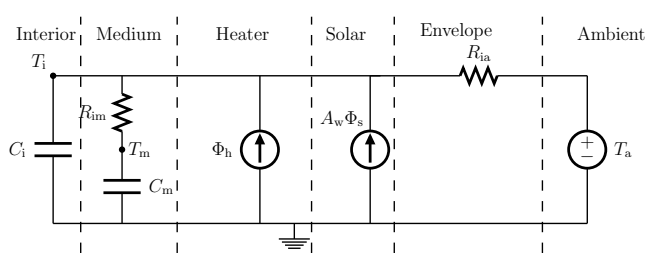

(b) RC-network network of TiTm.

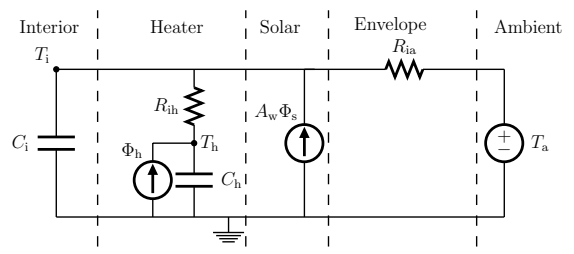

(d) RC-network network of TiTh.

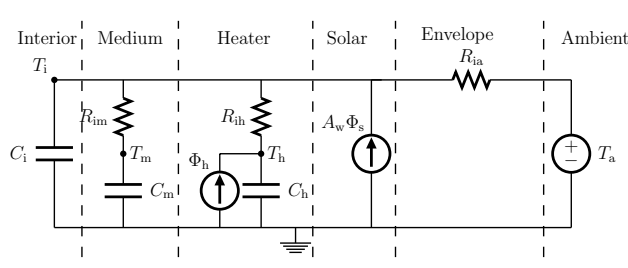

(f) RC-network network of TiTmTh.

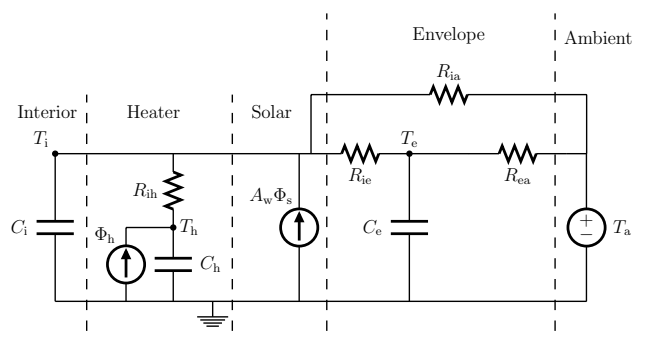

(h) RC-network network of TiTeThRia.

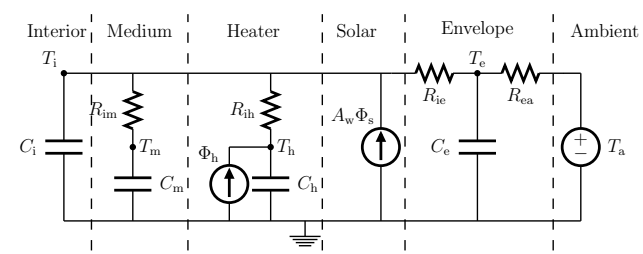

(j) RC-network network of TiTmTeTh. 


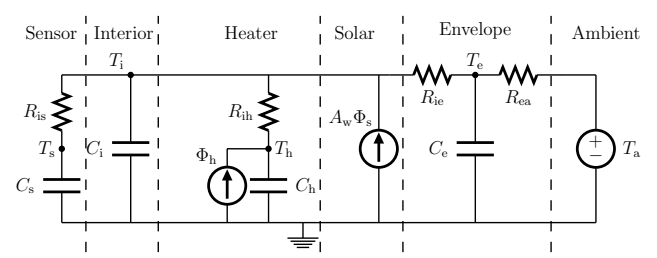

(k) RC-network network of TiTeThTs.

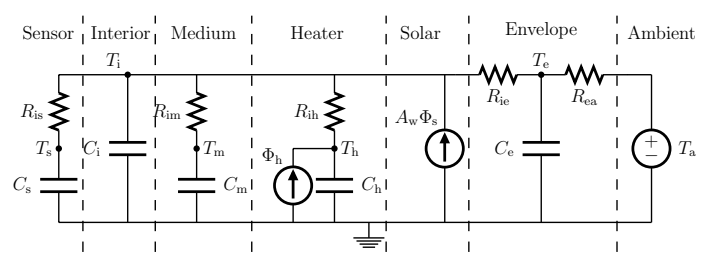

(m) RC-network network of TiTmTeThTs.

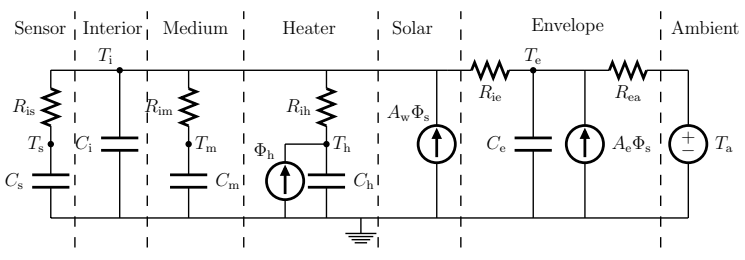

(o) RC-network network of TiTmTeThTsAe.

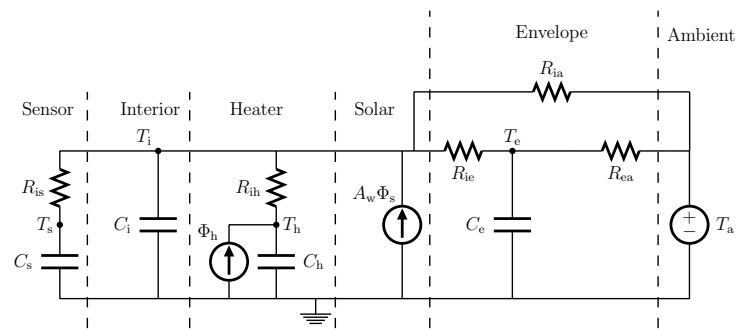

(l) RC-network network of TiTeThTsRia.

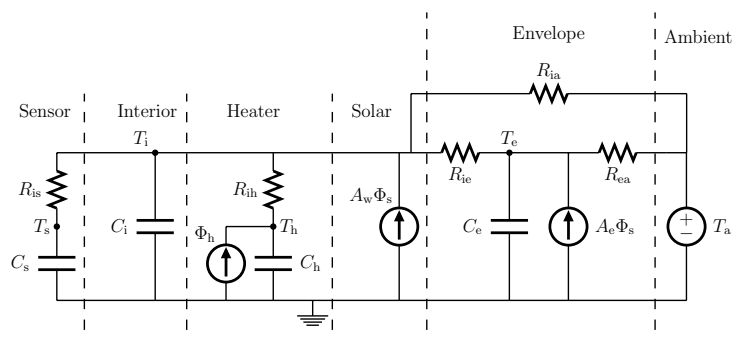

(n) RC-network network of TiTeThTsAeRia.

24 